\title{
La formación en periodismo científico desde la perspectiva del sistema nacional de I+D+i: el caso español
}

\author{
Training in scientific journalism from the perspective of the \\ national R\&D\&i system: the Spanish case
}

\author{
Formação em jornalismo científico a partir da perspectiva do sistema \\ nacional de I \& D: o caso espanhol
}

MARÍA DOLORES MENESES FERNÁNDEZ, Universidad de La Laguna, Canarias, España (dmeneses@ull.edu.es) YASMINA RIVERO ABREU, CEO Andrés Orozco, Canarias, España (yrivabr@gobiernodecanarias.org)

\section{RESUMEN}

La cultura científica tiene una importancia indudable. Una de las vías por las que conocemos los avances y las controversias científicas es el periodismo. Para saber si la formación periodística en Comunicación Social de la Ciencia y la Tecnología en los grados de Periodismo en España es adecuada, analizamos mediante una metodología exploratoria y cuantitativa- los planes de estudios y las guías docentes de las universidades españolas. Nuestras referencias han sido la estructura científico-tecnológica española y el gasto en I+D+i. Los resultados revelan unas dinámicas lógicas y otras paradójicas en la correlación entre formación y nivel científico nacional.

Palabras clave: comunicación de la ciencia; periodismo; I+D+i; cultura científica; ciencia y sociedad.

\section{ABSTRACT}

Nowadays, scientific culture has undoubted importance. Its advances and controversies are brought to us, among other ways, by journalism. To know if the journalistic formation in the social communication of science and technology in the studies of degree in journalism in Spain is pertinent, we analyzed, through an exploratory study and a quantitative methodology, their academic curriculum and their teaching guides, based on the scientific-technological Spanish structure and REDEI budgets. The results reveal some logical and other paradoxical dynamics in the correlation between training and regional scientific level.

Keywords: public communication of sciences; REDEI; journalism; scientific culture; science and society.

\section{RESUMO}

A cultura científica tem indubitável importância. Uma das maneiras pelas quais conhecemos os avanços e as controvérsias científicas é o jornalismo. Para saber se o ensino jornalístico na comunicação social de ciência e tecnologia na carreira universitária de licenciatura em Jornalismo na Espanhaé pertinente, analisamos com metodologia exploratória e quantitativa os planos de estudos e guias das unidades curriculares das universidades. Nossas referências foram a estrutura cientifico-tecnológica espanhola e os gastos em I\&D. Os resultados revelam uma dinâmica lógica e outra paradoxal na correlação entre formação e nível científico nacional.

Palavras-chave: comunicação da ciência; jornalismo; I\&D e inovação; cultura científica; ciência e sociedade.

Forma de citar:

Meneses Fernández, M. D. \& Rivero Abreu, Y. (2017). La formación en periodismo científico desde la perspectiva del sistema nacional de I+D+i: el caso español. Cuadernos.info, [41), 107-122. https://doi.org/10.7764/cdi.41.1145 


\section{INTRODUCCIÓN}

Tras diez años de implantación de los grados en Periodismo y cuarenta impartiendo las anteriores licenciaturas en Ciencias de la Información, la Comunicación y el Periodismo, la formación en Comunicación Social de la Ciencia y la Tecnología (CSCT) es una materia de estudio con presencia académica continuada en España. En este trabajo, exploramos las asignaturas de los grados en Periodismo, con base en el Sistema Español de Ciencia, Tecnología e Innovación (SECTI), para saber si existe correlación entre la inversión en I+D+i y la oferta formativa en CSCT de cada comunidad autónoma ${ }^{1}$.

Los estudiantes de Periodismo proceden, por lo general, del Bachillerato de Humanidades y Ciencias Sociales. Muchos de ellos perciben la CSCT como un campo complicado, relacionado con temáticas que suelen considerar ajenas a sus intereses académicos y profesionales. Por su parte, los profesores de Periodismo suelen incidir en aspectos de redacción -uso de tecnicismos y de mecanismos lingüísticos para aclararlos, y uso de los géneros periodísticos- por ser los recursos expresivos para traducir el lenguaje especializado al estándar del periodismo (Gutiérrez, 1998; Giraldo, 2015).

No obstante, junto con los contenidos sobre redacción periodística, han ido asentándose otros centrados en la estructura y agentes del sistema científico regional y nacional, ya que conviene que los futuros periodistas conozcan las diversas facetas de la I+D+i, dada su repercusión en el desarrollo social y en el bienestar de las personas.

\section{MARCO DE REFERENCIA}

Desde la década de los noventa del siglo XX, autores americanos y europeos trabajan en la consistencia de la CSCT. En 1994, Lewenstein revisó las tendencias de la cultura científica en Estados Unidos, lo que le llevó a proponer una definición. Hoy, este campo de investigación comunicológica abarca las acciones públicas impulsoras de la inmersión ciudadana en los asuntos de ciencia y de la penetración de la ciencia en la ciudadanía (citizen science). En 2016, Sánchez-Mora planteó una ordenación de la comunicación pública de la ciencia, de sus objetivos, medios y resultados, efectos y públicos. Dichas acciones expresan nuevos nexos de los ciudadanos con ámbitos altamente especializados y restringidos.

En este sentido, y a fin de ordenar la complejidad del fenómeno comunicativo al que se remite este trabajo, Bucchi (2008) compiló los diversos sentidos dados a la public communication of science en investigaciones teóricas y empíricas reconocibles en la literatura especializada, aportando un marco de trabajo multimodelo basado en el paradigma de comunicación imperante, en qué se enfatiza (contenido/contexto), en las versiones dominantes en la comunicación de la ciencia, en sus objetivos y en los contextos ideológicos de referencia. Esas acciones también pueden ser promovidas por ciudadanos desde fuera de los estamentos comunicativos profesionales; un ejemplo es el denominado discurshow (Mariño, 2016).

En esa línea de pensamiento cabe señalar que en los estudios de Periodismo de las facultades de Comunicación españolas domina la idea de que la especialización es prioritaria, quedando en segundo lugar en los criterios de calidad, solo por debajo de equipamientos y recursos (Centro ESADE de Industrias de la Cultura, 2008). Dada esa prioridad, interesa conocer qué sentido de la (pre)especialización en CSCT se extrae de la formación de grado.

\section{PREMISAS CONCEPTUALES DE PARTIDA}

Los autores entienden por comunicación social o pública de la ciencia y la tecnología el conjunto de estrategias difusoras y los formatos textuales y discursivos producidos profesionalmente, con el fin de informar a la ciudadanía, de forma legible, comprensible y aplicable, de los avances, descubrimientos y hallazgos logrados en las distintas disciplinas científicas y tecnológicas. Esas vías incluyen el periodismo, la divulgación, el marketing, la publicidad y las relaciones públicas. Comprenden toda acción comunicativa pública externa a los círculos científicos, en cuyo seno se redactan y publican los textos-fuente con lenguajes especializados propios de los cauces académicos. Estas acciones de la CSCT contribuyen a democratizar el conocimiento científico y tecnológico, y a generalizar la cultura científica entendida como constructo social y apropiación para la toma de decisiones individuales (Trelles \& Rodríguez, 2013). Podríamos decir que la CSCT es el conjunto de acciones comunicativas que favorece la inmersión de la ciencia en la ciudadanía y de los ciudadanos en la ciencia.

La CSCT así entendida vuelve permeables tres esferas: la investigadora, la política-científica y la empresarial, y facilita que el flujo de saberes científicos y tecnológicos trascienda más allá de donde se generan, actuando como motor de la transformación individual y social. La presencia de la CSCT en el discurso público depende, entre otros factores, de que las universidades 
desplieguen su función formativa periodística sobre el sistema investigador, la I+D+i y los agentes participantes en dicho sistema (Alcíbar, 2015). A esto se suma que la CSCT constituye un objeto de estudio para comunicólogos y para los propios científicos, cuyas investigaciones configuran el corpus teórico en el que instruir a los estudiantes de Periodismo.

En la exposición de motivos del título VII de la Ley Orgánica 6/2001, del 21 de diciembre, de Universidades se establece que la universidad tiene un papel de liderazgo en el proceso de transformación de los modos de generar y transmitir conocimientos derivados de la investigación científica y del desarrollo tecnológico. Se une que las enseñanzas de grado en Periodismo se organizan, en el marco del Espacio Europeo de Educación Superior (EEES), como titulación profesionalizante.

La especialización periodística por temáticas requiere que los estudiantes adquieran conocimientos conceptuales y competenciales. En periodismo científico, una buena capacitación profesional pasa por ser formados en el sistema científico, en sus agentes, y en las líneas de investigación priorizadas por la política científica según los nuevos retos en I+D+i que van surgiendo en una sociedad cambiante como la nuestra. Esta complejidad de base justifica que nos hayamos propuesto conocer el modo en el que están estructurados los programas formativos de los grados de Periodismo para identificar en ellos la formación orientada a la CSCT.

En el Libro Blanco de los Títulos de Grado en Comunicación (ANECA, 2005) se reservaron créditos obligatorios para la materia de Periodismo Especializado, destinándola a contenidos y competencias referidas al "estudio de la producción informativa de diferentes áreas de especialización periodística”. A partir de esa recomendación, cada universidad española desarrolló esta materia remitiéndola, de acuerdo a ese documento, a "los diferentes sistemas político, económico, cultural, de sociedad, deportivo, científico [...]". El objetivo fue garantizar que los estudiantes adquirieran la "capacidad y habilidad para el uso del argot y del conocimiento de las fuentes específicas y de los temas propios de la especialidad correspondiente".

Se reconoce así la necesidad de formar a los futuros periodistas en las temáticas científicas y tecnológicas. Desde la implantación de los estudios de grado, las universidades pueden diseñar una oferta de asignaturas optativas que diversifique la especialización, mediante itinerarios o no; es aquí donde se despliegan las asignaturas específicas de CSCT. En este punto surge el interés por analizar el nexo entre el desarrollo científico-tecnológico español y la formación universitaria en CSCT.

El EEES, además de otorgar a las titulaciones de grado un carácter profesionalizante, concibe la universidad inserta en la sociedad y en conexión con el mundo empresarial, que es la salida laboral natural de los egresados. Esta idea otorga protagonismo a los perfiles profesionales demandados por las organizaciones y amplía la visión universitaria al contemplar a las empresas como un agente colaborador en la formación de sus futuros empleados. De este modo, la formación de grado ha dado un giro sustancial al superarse la concepción de un aprendizaje basado en la acumulación de conocimientos disciplinares para priorizar la capacitación que facilite acceder al mercado laboral (Diezhandino, 2012; Rosique, 2013). Los perfiles profesionales periodísticos exigen tratar con fuentes expertas, documentos especializados y argot de los ámbitos de especialización (ANECA, 2005), así como conocer cómo están estructurados y cuál es el funcionamiento de esos ámbitos; uno de ellos es el sistema científico-tecnológico, esto es, la I+D+i.

Las especificidades del periodismo de $\mathrm{I}+\mathrm{D}+\mathrm{i}$ espolean el debate sobre a quiénes corresponde comunicar los logros de la ciencia y la tecnología. El tratamiento periodístico de estos temas no siempre ha satisfecho las expectativas de los investigadores (Meneses \& Martín, 2015). Periodistas y científicos difunden contenidos a uno y otro lado de la línea que diferencia la comunicación periodística de la divulgación científica; las acciones comunicativas de periodistas y de científicos divulgadores difieren en autoría, responsabilidad deontológica, enfoques, tratamientos, legibilidad, comprensibilidad, rigor, precisión, objetivos, intencionalidad, destinatarios, tipos de textos y canales.

De hecho, uno de los elementos distintivos del periodismo de I+D+i frente a la divulgación científica es el componente de servicio vinculado ética y deontológicamente al primero como profesión. Al periodista se le debe formar para que explique los beneficios de los avances científico-tecnológicos y las precauciones aconsejables ante las controversias inherentes a la investigación. Moreno (1999) y Méndez (2013) sostienen que el periodismo científico contribuye a que los ciudadanos entiendan los aspectos tecnocientíficos que les afectan, previniéndoles de los riesgos asociados. Esta visión contiene una de las caracterizaciones transversales del periodismo especializado: el componente de servicio, ya que ¿cómo se abordan acciones periodísticas 
con componente de servicio si no se posee dominio del tema para identificar dónde radica, precisamente, ese componente?

Estamos ante una nueva relación entre ciencia, periodismo, enseñanza superior y sociedad. Esa relación demanda más y mejor colaboración entre periodistas, científicos y docentes para mejorar la CSCT y la cultura científica de los ciudadanos. Tales fines requieren periodistas con una formación que les acredite como interlocutores fiables ante la comunidad científica. Esa formación precisa de asignaturas acordes con el escenario actual definido por el sistema ciencia y la I+D+i.

\section{NORMATIVA Y DESARROLLO CIENTÍFICO- TECNOLÓGICO EN ESPAÑA}

En las últimas décadas, dos leyes han regulado la actividad científica y tecnológica en España. La primera, la Ley 13/1986, de 14 de abril, de Fomento y Coordinación de la Investigación Científica y Técnica, luego sustituida por la Ley 14/2011, del 1 de junio, de la Ciencia, la Tecnología y la Innovación. Con esta norma, aun en desarrollo, España se dota de un marco organizativo en materia de ciencia y tecnología, uno de cuyos objetivos es apoyar la generación, difusión y transferencia del conocimiento que permitan resolver los desafíos de la sociedad.

Su artículo 3 alude al SECTI (CES, 2015), que integra las políticas públicas desarrolladas por la Administración General de Estado y por las comunidades autónomas. En él se contemplan tres tipos de agentes de coordinación y gestión, de ejecución (Art. 3.3 de la Ley 14/2011) y de financiación (CES, 2015).

Junto con el Estado, el sector privado constituye la otra fuente de financiación para generar conocimiento científico y tecnología, ya que los recursos materiales y humanos de las empresas permiten la explotación del conocimiento como activo de inversión (García, 2011). Incluyen los centros tecnológicos y los de apoyo a la innovación tecnológica del sector privado.

Otro aspecto que contempla la ley es la creación del Sistema de Información sobre Ciencia, Tecnología e Innovación, cuya finalidad es la captación de datos y análisis para la elaboración y seguimiento de la Estrategia Española de Ciencia y Tecnología y de Innovación 2013-2020 (EECTI), y de sus planes de desarrollo (MINECO, 2013).

En este entramado legislativo, la política científica española no reconoce la importancia de la labor de los agentes comunicadores - periodistas y medios- en la difusión científica y tecnológica. Sin embargo, la Ley
14/2011 sí solicita a los agentes del SECTI que asuman la difusión. En su Título III sobre Impulso de la investigación científica y técnica, la innovación, la transferencia del conocimiento, la difusión y la cultura científica, tecnológica e innovadora (art. 38.2.b-d), se insta a "fomentar la comunicación científica e innovadora por parte de los agentes del Sistema Español de Ciencia, Tecnología e Innovación", sin aludir a los medios de comunicación públicos ni a otros agentes difusores. Esta falta de concreción deja abierto el artículo 38.1, dedicado a la cultura científica y tecnológica, que dice:

Las Administraciones Públicas fomentarán las activi-
dades conducentes a la mejora de la cultura científica
y tecnológica de la sociedad a través de la educación,
la formación y divulgación, y reconocerán adecuada-
mente las actividades de los agentes del Sistema Espa-
ñol de Ciencia, Tecnología e Innovación en este ámbito.

Se incide en esta idea en los puntos j) y l) del artículo 2 de objetivos generales, y en los artículos 13.1 y 34.1.e). Esta insistencia en el fomento de la cultura científica y tecnológica enlaza con el hecho de ser considerada un indicador del desarrollo de las sociedades. A pesar de ello, lo que sigue primando son los parámetros económicos.

La recesión iniciada en 2008 ha debilitado la competitividad del país en innovación (MINECO, 2013) El informe Cotec (2014) también incide en sus consecuencias en el sistema español de innovación. En $2012^{2}$, los indicadores nacionales de I+D+i comenzaron a caer con intensidad. Ese año, el gasto español permanecía por debajo de los países de referencia de la UE-28 y de la Organización para la Cooperación y el Desarrollo Económicos (OCDE), una tendencia negativa acentuada por la disminución de la inversión nacional en ciencia desde 2009.

Esta disminución de inversión afectó por igual a administración, enseñanza superior y sector privado (CES, 2015), aunque el Ministerio de Economía y Competitividad afirma en las EECTI 2013-2020 que el número de doctores, investigadores y personal técnico dedicado a actividades de I+D+i se incrementó en España un 65\% en la última década (MINECO, 2013). El Ministerio atribuye estas cifras muy inferiores a las de la UE-28 y de los países de la OCDE a la escasa contratación de investigadores por parte de las empresas y a la falta de capacidad innovadora en los sectores productivos del país, cuyo bajo porcentaje de gasto empresarial en I+D+i lo sitúa a la cola del resto de países de la UE (MINECO, 2013). 
Otra medida de la capacidad científica y tecnológica de un país, región o institución son los indicadores bibliométricos. Los artículos científicos con filiación española en todas las áreas de conocimiento crecieron en 2012 un 4\% respecto de 2011, a pesar de reducirse la inversión en I+D+i. Este dato paradójico lleva a cuestionarse quién está asumiendo parte de los gastos de la investigación científica en España.

Un dato relevante aportado por el Índice Global de Innovación señala que la principal debilidad de España no es la producción de conocimiento, sino la capacidad del sistema de innovación español para transformarlo en resultados económicos (Cotec, 2014). Los avances quedan expuestos en el papel sin transformarse en resultados económicos. Ocurre algo similar con el número de patentes europeas e internacionales registradas por España.

La situación descrita sitúa al país en una posición complicada para progresar en los indicadores que miden la competitividad científica y tecnológica mundial. Solo en el índice del World Economic Forum de 2013 España ascendió una posición con respecto a 2012, si bien en la clasificación del Institute for Management Developement descendió cinco en un año.

La evolución de todos estos indicadores de la I+D+i española se plasma en el Mapa de Infraestructuras Científicas y Técnicas Singulares (ICTs) (MINECO, 2013, s.f.). España ha experimentado una importante apertura internacional en materia científica y tecnológica. Lo refleja las políticas diseñadas para corregir los datos negativos anteriores; ente ellas están la participación en programas y proyectos transnacionales, como el VII Programa Marco de la Unión Europea, y la adaptación al EEES y al Espacio Europeo de Investigación (EEI), impulsora de la reforma universitaria.

\section{FORMACIÓN EN COMUNICACIÓN CIENTÍFICA Y TECNOLÓGICA}

Una vez expuesta de manera sucinta la $\mathrm{I}+\mathrm{D}+\mathrm{i}$ nacional, corresponde revisar la situación de la formación universitaria en CSCT. En España se imparten grados en Periodismo en todas las comunidades autónomas salvo en Extremadura, La Rioja e Islas Baleares, y las ciudades autónomas de Ceuta y Melilla. La revisión realizada del Sistema Español de Ciencia y Tecnología y de la I+D+i permite saber cómo se relaciona con él la oferta formativa en CSCT.

El último informe sobre la Estrategia Nacional de Ciencia y Tecnología (ENCYT) alude a la trascendencia de comunicar los resultados científico-tecnológicos a la sociedad (FECYT, 2007). El periodista debe informar de resultados, pero también de cómo funciona la ciencia, de su gestión y de la política científica. La importancia de las estructuras comunicativas de las que disponemos para comprender la relevancia de la temática científico-tecnológica queda manifiesta en la cita "el conocimiento científico y técnico, su producción y su aplicación a todos los ámbitos de la vida de nuestras sociedades, es una de las fuerzas motrices de los procesos de crecimiento económico y de mejora del bienestar social" (FECYT, 2007).

En medio de normativas cambiantes sobre política educativa y científica para fomentar la I+D+i, la figura del periodista especializado en ciencia, salud, tecnología, biotecnología y medioambiente se diluye en el mar de atribuciones de los agentes investigadores, que no tienen la comunicación como misión ni como parte de su formación ni como salida profesional. Por ello, cabe preguntarse si en este escenario las universidades, las facultades de Comunicación Social y los periodistas ponen suficientemente en valor su posición óptima para asumir lo expuesto en la Ley de la Ciencia, la Tecnología y la Innovación. A este respecto, hace quince años Moreno $(2002,2003)$ puso de manifiesto el desfase al detectar que "los planes de estudios de periodismo adolecían de asignaturas con contenidos especializados en periodismo científico o de divulgación científica".

Lo anterior justifica que queramos relacionar la oferta formativa actual en CSCT de los grados en Periodismo con el sistema científico-técnico español, para averiguar cuántas titulaciones incluyen asignaturas de comunicación científica en sus planes de estudios y si estas abordan la I+D+i. De esta forma, conoceremos la adecuación de la formación y sabremos si se instruye a los estudiantes en la estructura e importancia del sistema científico-tecnológico. En definitiva, veremos si en los últimos años ha evolucionado la formación en periodismo de I+D+i impartido en el primer ciclo universitario.

El cambio inherente al sistema científico y el mercado laboral periodístico obliga a actualizar, con cierta frecuencia, la formación en aquellas competencias profesionales que exige, en este caso, la complejidad de la actividad investigadora. El componente de provisionalidad, incertidumbre, riesgo, polémicas y precaución que acompaña a los avances científicos, junto con los retos de la sociedad, deben estar contemplados en la formación periodística. De estarlo, quedarían reflejados en los planes de estudio y en las guías docentes de las asignaturas. 
Si bien ciertos autores reconocen que la especialización se desarrolla en posgrados (Rosique, 2013), entendemos que las asignaturas del grado en Periodismo son una vía introductoria para que los estudiantes descubran campos de especialización o se reafirmen en sus preferencias. Rosique subraya que el término competencia alude a varios grados de dominio temático que deberían adquirir los periodistas; en esa línea, consideramos que la preparación competencial se inicia en los estudios de grado. A pesar de la falta de consenso sobre cuál es la mejor formación del periodista, la comunidad académica internacional coincide en que estamos ante unos programas formativos con más convergencias que divergencias y ante un perfil profesional bien definido, con una función decisiva en la conformación de la realidad social (Fuente \& Mera, 2013). Facetas concretas de la formación, como el periodismo científicotecnológico, nos sitúan ante una capacitación tendente a la especialización, muchos de cuyos créditos se ofertan en los planes de estudios mediante las asignaturas optativas. Esto nos lleva a preguntarnos no solo en qué medida las universidades contemplan asignaturas de CSCT, sino si su oferta depende de pertenecer a una comunidad autónoma con mayor desarrollo en I+D+i.

\section{OBJETIVOS E HIPÓTESIS}

Los objetivos que nos planteamos fueron:

- O1. Conocer las asignaturas sobre comunicación científico-tecnológica de los planes de estudios del grado en Periodismo en España.

- O2. Averiguar si en las comunidades autónomas con una estructura de ciencia y tecnología más desarrollada, los grados en Periodismo de las universidades locales incluyen más asignaturas sobre CSCT respecto de otras autonomías.

- O3. Determinar en qué medida las asignaturas de CSCT instruyen en la organización, funcionamiento y producción del sistema científico y tecnológico nacional.

- O4. Estimar en qué medida las asignaturas de CSCT preparan, en cada universidad, en el sistema científico y en la producción científicotecnológica de la comunidad autónoma respectiva.

- O5. Conocer si en las asignaturas del grado en Periodismo se enseña la evolución diacrónica que ha conducido al panorama científico-tecnológico español actual.

Y, partiendo del binomio anunciado en el título de este estudio, las hipótesis fueron estas:

- H1. La implantación en España de los grados en Periodismo y el afianzamiento de la normativa sobre ciencia, tecnología e innovación y de los planes nacionales y regionales de I+D+i han fomentado la presencia de asignaturas sobre CSCT.

- H2. Las comunidades autónomas con un mayor desarrollo e inversión en I+D+i prestan mayor atención a la formación en CSCT, incluyendo al menos una asignatura sobre esta temática en los grados en Periodismo impartidos en su territorio.

- H3. En las guías docentes de las asignaturas se contempla formar en la realidad científicotecnológica del país, y en el Sistema Español de Ciencia, Tecnología e Innovación.

- H4. En las guías docentes de las asignaturas se contempla formar en producción científica y tecnológica del país y de la comunidad autónoma a la que pertenece cada universidad.

\section{FUENTES Y METODOLOGÍA}

Por una parte, identificamos las universidades en la web del Registro de Universidades, Centros y Títulos (RUCT) del Ministerio de Educación, Cultura y Deporte del Gobierno de España. El RUCT nos remitió a las webs de las universidades con grado en Periodismo, dado que la normativa sobre transparencia informativa garantiza que estos portales difunden los planes de estudios y las guías docentes vigentes. Ambos documentos - guías docentes y planes de estudios publicados en el Boletín Oficial del Estado-conforman las fuentes oficiales consultadas. Si hubo necesidad, se consultó la memoria de verificación del grado. Revisamos las 37 universidades con estudios de Periodismo de las 85 que conforman el sistema educativo superior español (50 públicas y 35 privadas).

Puesto que las titulaciones no cambian su plan de estudios de un año académico a otro (requiere un proceso de modificación), centramos nuestro análisis en los documentos académicos de 2014-2015. Por tanto, consideramos metodológicamente válido focalizarnos en ese curso. Una vez localizados los documentos académicos 
de los grados en Periodismo, rastreamos en ellos las asignaturas (variable 1 en este estudio) cuya denominación las vinculara con la CSCT. En la búsqueda incluimos las asignaturas denominadas Periodismo Especializado por la posibilidad de que contemplaran contenidos de interés en este estudio; así, en el caso de que un epígrafe del temario de la guía de esta asignatura se dedicara a algún aspecto de la CSCT se le asignó el valor 1; de aparecer varios subepígrafes, se dividió la unidad (1) entre el número de subepígrafes para ponderar el valor cuantitativo de la presencia de dichos contenidos. Los datos obtenidos se registraron en una ficha que luego volcamos en una aplicación informática para cruzarlos. Los resultados se sintetizaron en tablas y gráficos.

Por otra parte, las fuentes consultadas para obtener los datos del panorama científico y tecnológico de las comunidades autónomas (variable 2 en este estudio) fueron, como se deduce del epígrafe 2 anterior, el libro de Indicadores del Sistema Español de Ciencia y Tecnología y el Mapa de las Instituciones de I+D+i, elaborados por el Observatorio Español de I+D+i de la Fundación Española para la Ciencia y la Tecnología (FECYT, 2014). Los indicadores seleccionados, en coherencia con nuestros objetivos e hipótesis, incluyeron la inversión económica, la inversión en capital humano, instituciones e infraestructuras.

Estas fuentes documentales oficiales desagregan los indicadores por comunidades autónomas, facilitándonos correlacionarlos con el desarrollo científicotecnológico y con la formación en CSCT de los grados de Periodismo de cada autonomía.

\section{RESULTADOS}

La muestra resultó integrada por 27 asignaturas, de ellas 19 están relacionadas con la CSCT y otras ocho son de Periodismo Especializado con parte de sus contenidos dedicados a esta temática. Pasamos a analizar la correspondencia entre los datos académicos con los del desarrollo científico-tecnológico de las comunidades autónomas.

\section{UNIVERSIDADES CON ASIGNATURAS DE CSCT EN EL GRADO EN PERIODISMO}

El periodismo de ciencia, tecnología, salud y medioambiente está incluido en los planes de estudios de los grados de dos formas. Una es limitándolo a uno o varios temas en la asignatura obligatoria de Periodismo Especializado, a modo de formación introductoria. Otra es ofreciendo optativas específicas de CSCT; en este caso diferenciamos entre universidades con una o con más asignaturas específicas (tabla 1).

En el curso del que hemos realizado este estudio (2014-2015), 37 universidades públicas y privadas españolas impartían el grado en Periodismo; de esas, 22 incluyen en el plan de estudios contenidos de CSCT. Las 15 restantes ni siquiera contemplan temas relacionados con ciencia y tecnología en la asignatura de Periodismo Especializado (tabla 1). Esto implica que el 40,5\% de los grados no inicia a sus estudiantes en esta temática.

De las 27 asignaturas analizadas en 22 grados, 19 (70,4\%) están dedicadas a CSCT y ocho $(29,6 \%)$ asignaturas de Periodismo Especializado dedican parte de su temario a ciencia y tecnología. Así, cuatro universidades imparten más de una asignatura relacionada con la CSCT; diez imparten una asignatura relacionada con la CSCT; ocho incluyen Periodismo Especializado con temas de ciencia y tecnología; una ofrece Periodismo Especializado sin contenidos especificados y 16 carecen de asignaturas relacionadas con la CSCT.

Cuatro (10,8\%) universidades públicas de las 37 (Murcia, Valencia, Málaga y Complutense de Madrid) incluyen en sus grados en Periodismo más de una asignatura de CSCT; 10 (27\%) imparten una. Esos cuatro grados de Periodismo con varias asignaturas (hasta cuatro) sobre esta temática son los que más enfatizan la formación en CSCT, sobresaliendo en el conjunto de universidades españolas. Dedicar al menos una optativa a esta temática garantiza que una parte de los egresados posea competencias en CSCT, facilitándoles continuar especializándose en estudios de posgrado.

Analizamos también la concordancia entre comunidades autónomas, universidades, grados y formación en CSCT (tabla 2), para luego correlacionar los resultados con la inversión regional en I+D+i.

Las comunidades autónomas de Madrid y Cataluña registran los porcentajes más altos de asignaturas de CSCT en los grados de Periodismo, ya que concentran el mayor número de universidades en su territorio. Les siguen Murcia, Castilla y León y Andalucía³. En la región de Murcia, una de las dos universidades con grado en Periodismo (Universidad de Murcia) ofrece una asignatura obligatoria (Ciencia, Tecnología y Sociedad Global en el siglo XXI), más tres optativas (Periodismo sobre Salud y Medioambiente, Periodismo sobre Ciencia y Tecnología, y Comunicación Divulgativa), más la obligatoria de Periodismo Especializado con un tema dedicado a información científica; esta titulación supone un caso excepcional en el conjunto de universidades españolas, destacando a la región de Murcia -con tres universidades, una de las cuales es politécnica-si la comparamos con Madrid y Cataluña. 


\begin{tabular}{|c|c|c|c|}
\hline $\mathrm{N}^{0}$ & Universidad & Asignaturas de CSCT & Comunidad Autónoma \\
\hline 1 & de Valencia & Más de una asignatura & Valencia \\
\hline 2 & Complutense de Madrid & Más de una asignatura & Madrid \\
\hline 3 & de Murcia & Más de una asignatura & Murcia \\
\hline 4 & de Málaga & Más de una asignatura & Andalucía \\
\hline 5 & de Valladolid & Una & Castilla y León \\
\hline 6 & de Zaragoza & Una & Aragón \\
\hline 7 & Carlos III de Madrid & Una & Madrid \\
\hline 8 & Europea de Madrid & Una & Madrid \\
\hline 9 & Camilo José Cela & Una & Madrid \\
\hline 10 & Miguel Hernández, Elche & Una & Valencia \\
\hline 11 & Pompeu Fabra & Una & Cataluña \\
\hline 12 & de La Laguna & Una & Canarias \\
\hline 13 & de Sevilla & Una & Andalucía \\
\hline 14 & Autónoma de Barcelona & Una & Cataluña \\
\hline 15 & Católica San Antonio & Periodismo Especializado & Murcia \\
\hline 16 & Antonio de Nebrija & Periodismo Especializado & Madrid \\
\hline 17 & del País Vasco & Periodismo Especializado & País Vasco \\
\hline 18 & Europea Miguel de Cervantes & Periodismo Especializado & Castilla y León \\
\hline 19 & Rovira i Virgili & Periodismo Especializado & Cataluña \\
\hline 20 & Abat Oliba CEU & Periodismo Especializado & Cataluña \\
\hline 21 & San Pablo-CEU & Periodismo Especializado & Madrid \\
\hline 22 & San Jorge & $\begin{array}{l}\text { Guía docente Periodismo } \\
\text { Especializado sin contenidos }\end{array}$ & Aragón \\
\hline
\end{tabular}

Tabla 1. Universidades con asignaturas de CSCT en su grado en Periodismo

Fuente: Elaboración propia.

\begin{tabular}{lccc}
$\begin{array}{l}\text { Comunidades } \\
\text { autónomas }\end{array}$ & $\begin{array}{l}\mathrm{N}^{0} \text { de universidades con } \\
\text { asignaturas de CSCT }[23)\end{array}$ & $\begin{array}{l}\mathrm{N}^{0} \text { de universidades con } \\
\text { grado en Periodismo (28) }\end{array}$ & $\begin{array}{c}\text { Reparto porcentual } \\
\text { Madrid }\end{array}$ \\
\hline Cataluña & 4 & 7 & $27 \%$ \\
\hline Valencia & 2 & 3 & $18 \%$ \\
\hline Murcia & 2 & 2 & $10 \%$ \\
\hline Castilla y León & 2 & 2 & $10 \%$ \\
\hline Andalucía & 2 & 3 & $10 \%$ \\
\hline Aragón & 2 & 2 & $10 \%$ \\
\hline País Vasco & 1 & 2 & $5 \%$ \\
\hline Canarias & 1 & 1 & $5 \%$ \\
\hline
\end{tabular}

Tabla 2. Reparto autonómico de universidades con asignaturas de CSCT

Fuente: Elaboración propia.

CUADERNOS.INFO Nº 41 / DICIEMBRE 2017 / ISSN 0719-3661 / Versión electrónica: www.cuadernos.info / ISSN 0719-367X 


\section{Asignatura}

\begin{tabular}{|c|c|c|}
\hline Periodismo Especializado en Ciencia y Salud & 5 & 5 \\
\hline Periodismo Especializado & 10 & 10 \\
\hline P. de Ciencia y Tecnología y su tratamiento & 7 & 7 \\
\hline Periodismo Especializado 2 & 4 & 33 \\
\hline Periodismo Científico y Medioambiental & 9 & 9 \\
\hline Periodismo Científico y Medioambiental & 4 & 4 \\
\hline Periodismo Especializado & 1 & 10 \\
\hline Periodismo Científico & 5 & 5 \\
\hline Periodismo Cultural y Científico & 3 & 5 \\
\hline Periodismo Científico y Ambiental & 7 & 7 \\
\hline Periodismo sobre Salud y Medioambiente & 7 & 7 \\
\hline P. Especializado en Ciencia y Cultura & 1 & 10 \\
\hline P. Especializado: Ciencia, Economía y Deportes & 1 & 4 \\
\hline Periodismo Científico y Medioambiental & 9 & 9 \\
\hline Periodismo Científico y Medioambiental & 5 & 5 \\
\hline Periodismo Especializado 2 & 3 & 13 \\
\hline Periodismo Especializado 2 & 1 & 3 \\
\hline Com. Biomédica, Tecnológica y Medioambiental & 9 & 9 \\
\hline Periodismo Especializado & 1 & 5 \\
\hline Divulgación de Inf. Científica y Tecnológica & 0 & 0 \\
\hline Periodismo sobre Ciencia y Tecnología & 6 & 6 \\
\hline P. Especializado en Economía y Medioambiente & 5 & 10 \\
\hline Comunicación Divulgativa & 12 & 12 \\
\hline Periodismo Especializado & 1 & 10 \\
\hline Teoría y práctica del P. Especializado & 1 & 11 \\
\hline Comunicación y Salud & 14 & 14 \\
\hline Periodismo Científico y Medioambiental & 4 & 4 \\
\hline
\end{tabular}

Tabla 3. Comparativa entre el número total de temas de cada asignatura y los dedicados a CSCT

P. $=$ Periodismo, Com.$=$ Comunicación, Inf. $=$ Información

Fuente: Elaboración propia

\section{TIPOLOGIAS DE ASIGNATURAS Y CONTENIDOS}

En el conjunto de las universidades, de las 27 asignaturas dedicadas a CSCT 15 son obligatorias (54\%) y 12 optativas (46\%). Las tablas 3 y 4 comparan el total de temas de las 27 asignaturas analizadas y los dedicados, de ellos, a CSCT. Dado que no todos los temas de la asignatura obligatoria de Periodismo Especializado ni, de hecho, de otras se ocupan de esta temática, procede averiguar qué parte del temario se le destina.

Tras analizar el temario de las 27 asignaturas, 


\begin{tabular}{|c|c|c|}
\hline Asignatura (aglutinadas por denominación) & Universidad & Comunidad autónoma \\
\hline P. E. en Ciencia y Salud & Pompeu Fabra & Cataluña \\
\hline \multirow{8}{*}{ Periodismo Especializado } & Abat Oliba CEU & Cataluña \\
\hline & Antonio de Nebrija & Madrid \\
\hline & Católica San Antonio de Murcia & Región de Murcia \\
\hline & Europea Miguel de Cervantes & Castilla y León \\
\hline & de La Laguna & Canarias \\
\hline & del País Vasco & País Vasco \\
\hline & Rovira i Virgili & Cataluña \\
\hline & San Pablo CEU & Andalucía \\
\hline P. de Ciencia y Tecnología y su Tratamiento & de Valencia & Valencia \\
\hline \multirow{3}{*}{ Periodismo Especializado 2} & San Pablo CEU & Andalucía \\
\hline & Abat Oliba CEU & Cataluña \\
\hline & Antonio de Lebrija & Madrid \\
\hline \multirow{4}{*}{ Periodismo Científico y Medioambiental } & Miguel Hernández de Elche & Valencia \\
\hline & Europea de Madrid & Madrid \\
\hline & Carlos III & Madrid \\
\hline & Camilo José Cela & Madrid \\
\hline \multirow{2}{*}{ Periodismo Científico } & Autónoma de Barcelona & Cataluña \\
\hline & de Zaragoza & Aragón \\
\hline Periodismo Cultural y Científico & de Valladolid & Castilla y León \\
\hline Periodismo Científico y Ambiental & de Sevilla & Andalucía \\
\hline Periodismo sobre Salud y Medioambiente & de Murcia & Región de Murcia \\
\hline P. Especializado en Ciencia y Cultura & Complutense de Madrid & Madrid \\
\hline P. E. Ciencia, Economía y Deportes & Católica San Antonio & Murcia \\
\hline Com. Biomédica, Tecnológica y Medioambiental & de La Laguna & Canarias \\
\hline Divulgación de Inf. Científica y Tecnológica & Valencia & Valencia \\
\hline Periodismo sobre Ciencia y Tecnología & de Murcia & Región de Murcia \\
\hline P. E. en Economía y Medioambiente & Complutense de Madrid & Madrid \\
\hline Comunicación Divulgativa & de Murcia & Región de Murcia \\
\hline \multirow{2}{*}{ Teoría y práctica del P. E. } & Autónoma de Barcelona & Cataluña \\
\hline & de Málaga & Andalucía \\
\hline Comunicación y Salud & Autónoma de Barcelona & Cataluña \\
\hline
\end{tabular}

Tabla 4. Filiación entre asignaturas y universidades

P. $=$ Periodismo, P. E. $=$ Periodismo Especializado, Com. $=$ Comunicación, Inf. $=$ Información

Fuente: Elaboración propia.

CUADERNOS.INFO Nº 41 / DICIEMBRE 2017 / ISSN 0719-3661 / Versión electrónica: www.cuadernos.info / ISSN 0719-367x 
obtuvimos que $16(59,3 \%)$ se centran completamente en CSCT. Las 11 (40,7\%) restantes lo dedican solo en parte; de estas, dos orientan más de la mitad del temario a ciencia y tecnología. La producción bibliográfica científica y tecnológica nacional solo aparece en los contenidos de 10 de las 27 asignaturas, aun tratándose de un indicador observado por las universidades.

En tres asignaturas, los temarios incluyen epígrafes dedicados a aspectos organizativos de la ciencia y la tecnología en España, como es el caso del Sistema Español de Ciencia, Tecnología e Innovación. Se observa, por tanto, que los aspectos organizativos y normativos de la ciencia y la tecnología no suelen estar presentes en la formación dada a los estudiantes, frente a otros de cariz periodístico-redaccional. Este dato, siendo objetivo, queda sujeto a interpretación debido a que las denominaciones de los epígrafes de los temarios solo anuncian lo que se trabajará en la asignatura. En cuanto a la historia de la ciencia y la tecnología, y a la difusión mediática de sus hitos, solo aparecen en una asignatura.

\section{COMPARATIVA ENTRE INVERSIÓN AUTONÓMICA EN I+D+I, GRADO EN PERIODISMO Y ASIGNATURAS DE CSCT}

Las nueve regiones con mayor inversión en 2012 (última anualidad con datos disponibles) fueron Madrid, Cataluña, Andalucía, País Vasco, la Comunidad Valenciana, Castilla y León, Galicia, Navarra y Aragón. Debe recordarse que las comunidades autónomas con más población reciben mayor presupuesto en el reparto nacional, convirtiéndose en las de mayor potencial para invertir en $\mathrm{I}+\mathrm{D}+\mathrm{i}$.

Murcia queda relegada del citado grupo de nueve autonomías; a pesar de su inversión menor en I+D+i, que depende del PIB regional (FECYT, 2014), es una región que da gran importancia a la formación en CSCT. Le acompaña, a distancia, Canarias. Las comunidades con más gasto son Madrid y Cataluña; pero la mayor inversión en términos porcentuales en relación al PIB corresponde al País Vasco y Navarra, cuyo liderazgo desplaza a Madrid y Cataluña. A este respecto, los datos aportados por los indicadores del Sistema Español de Ciencia, Tecnología e Innovación (FECYT, 2014), que comparan los euros invertidos en relación al número de habitantes de la región, revelan que la primacía es del País Vasco, seguido de Navarra y Madrid, y más alejada se sitúa Cataluña.

Teniendo en cuenta la inversión en ciencia y tecnología y la formación periodística, los datos revelan que las comunidades autónomas con asignaturas de CSCT en sus grados en Periodismo se correlacionan con las que destinan mayor inversión a I+D+i. Esta correlación no se cumple en Murcia ni en Canarias, ya que quedando excluidas de las diez primeras autonomías con mayor inversión, destacan académicamente en formación sobre CSCT.

Además, al contrario de lo que podría esperarse, no son las comunidades con mayor presupuesto en el reparto nacional (Madrid y Cataluña) las que hacen un mayor esfuerzo inversor en I+D+i en relación al PIB y al número de habitantes, sino que lo hacen el País Vasco y Navarra. Por ello es significativo que no se contemple en Navarra la formación en CSCT en sus grados en Periodismo 4 .

En general, las nueve comunidades en las que se imparten asignaturas de CSCT coinciden con las que tienen mayor número de centros I+D+i (Cataluña, Madrid y la Comunidad Valenciana, según FECYT, 2014 y MINECO, s.f.). Si en vez de centros I+D+i observamos los institutos de investigación mixtos, quedan excluidas del grupo de nueve Murcia y Canarias; y Murcia, Aragón y el País Vasco, si discriminamos por institutos universitarios. Cinco de las nueve comunidades con formación en CSCT son las primeras en inversión de capital y recursos humanos en $\mathrm{I}+\mathrm{D}+\mathrm{i}$, así como en número de institutos de investigación y estructuras de vanguardia investigadora en su territorio.

Veamos qué ocurre en el caso de los Parques Científicos y Tecnológicos (PCT) - proyectos asociados a un espacio físico que actúa como polo de desarrollo industrial en el que surgen empresas tecnológicas innovadoras-. Las nueve comunidades autónomas con formación periodística en CSCT vuelven a ser las primeras en número de PCT. En el caso de los organismos públicos de investigación (OPI), estos se concentran en Madrid, salvo uno con sede en Canarias (Instituto Astrofísico).

Respecto de los Centros Tecnológicos (CT) -entidades sin ánimo de lucro destinadas a contribuir al beneficio general de la sociedad, y a mejorar la competitividad del sector empresarial mediante el conocimiento tecnológico y actividades de I+D+i-, las comunidades autónomas con asignaturas de CSCT forman parte del grupo de las nueve con más CT, exceptuando Canarias que no aparece en el registro de CT de 2017 del Ministerio de Economía y Competitividad.

Considerando la distribución regional de los 10 tipos de instituciones de $\mathrm{I}+\mathrm{D}+\mathrm{i}$-incluidos los organismos de salud pública y las Oficinas de Transferencia de Resultados de la Investigación-, en términos globales, las 
autonomías de Cataluña, Madrid, la Comunidad Valenciana, Andalucía, Castilla y León, Aragón, Canarias, el País Vasco y la región de Murcia son las que acogen en su territorio la mayor parte de las instituciones científico-tecnológicas de España. Aun quedando Murcia rezagada, es la comunidad autónoma con la titulación de Periodismo que ofrece más asignaturas (cuatro) dedicadas a CSCT, una obligatoria y tres optativas.

\section{CONCLUSIONES}

La correlación que hemos establecido entre los resultados de la formación en CSCT y la I+D+i nacional y regional nos conduce a las conclusiones siguientes.

1. Hemos analizado los factores que inciden en la formación en CSCT de los grados en Periodismo de las universidades españolas, cumpliendo con el Ol de conocer las asignaturas sobre comunicación científico-tecnológica de los planes de estudios del grado en Periodismo en España. Esto ha permitido determinar en qué medida dichos planes incluyen asignaturas sobre CSCT, y con qué carácter (obligatorio u optativo) fueron diseñadas las dedicadas enteramente a CSCT (19 o 70\%) y las que le reservan una parte del temario (ocho o 30\%). Además, se identificó el reparto geográfico de las universidades con titulaciones que imparten estas asignaturas, comprobándose cuántas son en relación al total de universidades en España con grado en Periodismo y por comunidad autónoma. Esto permitió comprobar que Madrid reúne más universidades con asignaturas dedicadas plena o parcialmente a formar en CSCT. Le siguen Cataluña, Valencia, la región de Murcia, Castilla y León, Andalucía, Aragón, el País Vasco y Canarias.

Hemos verificado que la mayor concentración de titulaciones en las comunidades más potentes no supone que concedan más importancia a la CSCT, a pesar de que su nivel de desarrollo y presupuestos económicos estén muy por encima de las de menor población y asignación presupuestaria pública. Así, comunidades de menor tamaño y desarrollo destacan, respecto de otras mayores, por la oferta formativa en CSCT. Es el caso citado de la región de Murcia, que sobresale con cuatro asignaturas en el grado en Periodismo de la Universidad de Murcia, más parte del temario de la asignatura obligatoria Periodismo Especializado.
En general, destaca la asignatura obligatoria Periodismo Especializado con temas dedicados a CSCT, ya que la mayor parte de las asignaturas destinadas íntegramente a esta temática son optativas, salvo excepciones.

2. Existe una correlación entre las comunidades con mayor desarrollo en I+D+i y mayor inversión presupuestaria, y la oferta en sus universidades de asignaturas de CSCT. Este resultado contribuye a cumplir el $\mathrm{O} 2$ de averiguar si en las comunidades autónomas con una estructura científica y tecnológica más desarrollada los grados en Periodismo de las universidades locales incluyen más asignaturas sobre CSCT respecto de otras regiones.

3. Hemos cumplido el O3 de determinar en qué medida las asignaturas de CSCT instruyen en la organización, funcionamiento y producción del sistema científico y tecnológico nacional, y el O4 de estimar en qué medida esas asignaturas preparan, en cada universidad, sobre el sistema científico y en la producción científico-tecnológica de la comunidad autónoma respectiva, ya que pudimos identificar las que abordan la producción bibliográfica nacional; es el caso de Comunicación Divulgativa de la Universidad de Murcia, que incluye en su temario contenidos sobre las revistas académicas y los índices para medir su impacto científico. Sin embargo, ninguna asignatura alude a la producción científica autonómica, a pesar de constituir una fuente para los periodistas locales y foráneos.

4. Hemos cumplido el $\mathrm{O} 5$ de conocer si en las asignaturas del grado en Periodismo se enseña la evolución diacrónica que explica el panorama científico-tecnológico español actual, ya que hemos averiguado que en buena parte de las guías docentes no se incluyen contenidos formativos sobre la organización del Sistema Español de Ciencia, Tecnología e Innovación ni sobre la evolución histórica de la ciencia y cómo se ha difundido a lo largo del tiempo, a pesar de disponerse de publicaciones al respecto. Los casos que sí los contemplan son aislados (Universidad de La Laguna). Por su parte, la organización del Sistema Español de Ciencia, Tecnología e Innovación solo está presente en asignaturas impartidas en las universidades Pompeu Fabra, de Valencia y Carlos III. 
5. La Hl (la implantación en España de los grados en Periodismo y el afianzamiento de la normativa sobre ciencia, tecnología e innovación, y de los planes nacionales y regionales de I+D+i han fomentado la presencia de asignaturas sobre CSCT) queda refutada en la medida en que no todas las universidades españolas con grado en Periodismo imparten asignaturas de CSCT. Resulta complejo proponer razones que lo expliquen. Solo se ha podido determinar que, habitualmente, las que ofrecen estas asignaturas son las comunidades con un mayor desarrollo de la I+D+i y una mayor inversión presupuestaria.

6. Esta correlación verifica la H2 (las comunidades autónomas con un mayor desarrollo e inversión en I+D+i prestan mayor atención a la formación en CSCT, incluyendo al menos una asignatura sobre esta temática en los grados en Periodismo impartidos en su territorio)

7. Se observa, por tanto, una clara concomitancia entre el tamaño, la disponibilidad de recursos y el desarrollo científico de las comunidades autónomas, y la importancia que prestan sus universidades a la formación en CSCT.

Por lo anterior, comunidades con menos recursos, alejadas de los primeros puestos en inversión en I+D+i y desarrollo económico, como la región de Murcia y Canarias, sobresalen al conceder una importancia destacable a esta formación respecto de las comunidades con un sistema científicotecnológico más complejo e inversiones mayores.

8. La H3 (en las guías docentes de las asignaturas se contempla formar en la realidad científicotecnológica del país, y en el Sistema Español de Ciencia, Tecnología e Innovación) queda refutada por las razones expuestas en los puntos 2 y 5 de estas conclusiones.

9. Se refuta también la H4 (en las guías docentes de las asignaturas se contempla formar en producción científica y tecnológica del país y de la comunidad autónoma a la que pertenece cada universidad) por lo señalado en el punto 3 .

Se concluye que, a pesar de que la adaptación de las titulaciones al EEES provee de mecanismos para que las universidades adecuen sus planes de estudios a la realidad social, existen desajustes entre la formación en CSCT y la I+D+i española. Esos desajustes influyen, a corto plazo, en la formación de los periodistas y, a mediano y largo plazo, en la calidad del periodismo de ciencia y tecnología y de la cultura científica de los ciudadanos. Por lo tanto, cabría otorgar mayor presencia al sistema científico nacional en los grados en Periodismo. De no impartirse asignaturas específicas, el margen de maniobra para incluirlo en la formación de los estudiantes depende de los docentes que imparten materias afines.

\section{DISCUSIÓN}

Los resultados obtenidos son ilustrativos y diagnostican una situación formativa mejorable. No obstante, si retomamos la noción de CSCT que propusimos en el marco de referencia, este trabajo presenta ciertas limitaciones. Por ejemplo, si bien el testeo preliminar realizado de dobles grados de Periodismo más otra titulación muestra que sus asignaturas relacionadas con la CSCT suelen ser las mismas que las impartidas en el grado [simple] de Periodismo de la misma universidad, cabría revisar los dobles y triples grados de forma exhaustiva para corroborar o corregir esta idea. Esa revisión debería abarcar también los posgrados de Comunicación (másteres).

Resulta complejo estimar cuál es la formación más adecuada en CSCT en el primer ciclo. Los contenidos podrían ser los mencionados en este trabajo, y el marco deseable sería una asignatura optativa planteada como continuidad de la obligatoria de Periodismo Especializado.

En cualquier caso, la situación expuesta aquí revela que la formación en periodismo científico y tecnológico de grado requiere ser fomentada, cuando no reformulada, para que los egresados posean un cierto dominio del sistema I+D+i español, adecuado a su complejidad e importancia. Los contenidos impartidos reflejan que el Sistema Español de Ciencia y Tecnología y los sectores productivos relacionados con la I+D+i no terminan de generalizarse y consolidarse como un espacio de desenvolvimiento natural y cercano a los intereses de docentes y estudiantes de periodismo, a pesar de que sea una vía de proyección laboral para los egresados.

Queda por determinar si la concomitancia detectada entre sistema-ciencia, inversión en I+D+i y formación en CSCT es casual, si deriva de inercias de las antiguas licenciaturas o si obedece a decisiones tomadas durante el diseño de los grados en Periodismo en función del 
contexto científico y tecnológico regional y general. Saberlo requiere prolongar este estudio para explicar las ausencias y las excepciones detectadas. Ello contribuirá a entender mejor las causas del déficit formativo en la temática científico-tecnológica de los periodistas y de la baja cultura científica en España respecto de la Unión Europea (Fundación BBVA, 2012; López Cerezo y Cámara, 2009).
Mientras en periodismo se da esta situación, en Internet proliferan sitios web con notoria calidad, administrados por egresados de titulaciones diversas (Biología, Física, Historia, Ingenierías, Medicina) cuyas elevadas competencias científicas y habilidades comunicativas les posibilita difundir, de forma didáctica y entretenida, los avances y las controversias de las ciencias, las tecnologías y el medioambiente.

NOTAS

1. España se rige por un sistema de distribución de competencias entre las diversas Administraciones Públicas. Salvo las competencias que se reserva el Estado (artículo 149 de la Constitución Española de 1978), en las demás, las 17 comunidades autónomas (regiones) y las dos ciudades autónomas desempeñan su papel legislador, ejerciendo el autogobierno; así ocurre en materia de educación y universidades.

2. Años entre el Plan Nacional 2008-2011 y el de 2013-2016. Los datos estadísticos más recientes disponibles corresponden a 2012. Consideramos que constituyen un contexto próximo para dimensionar las guías docentes revisadas del curso 2014-2015.

3. Las dos universidades de Andalucía revisadas, de Málaga y de Sevilla, incluyen los centros adscritos, puesto que deben impartir idéntico plan de estudios y programa formativo.

4. Las asignaturas optativas sobre comunicación médica y fundamentos del periodismo científico, de la Universidad de Navarra, se imparten respectivamente en el grado de medicina, y en los grados de comunicación audiovisual y de publicidad y relaciones públicas.

\section{REFERENCIAS}

Alcíbar, M. (2015). Comunicación pública de la ciencia y la tecnología: una aproximación crítica a su historia conceptual [Public Communication of Science and Technology: a Critical Approach to its Conceptual History]. Arbor, 191(773), 1-13. https://doi.org/10.3989/arbor.2015.773n3012

ANECA (2005). Libro Blanco. Títulos de Grado en Comunicación [White book. Degree in Communication]. Madrid: Agencia Nacional de Evaluación de la Calidad y Acreditación.

Bucchi, M. (2008). Of deficits, deviations and dialogues: theories of public communications of sciences In M. Bucchi \& B. Trench (Ed.), Handbook of Public Communication of Science and Technology (pp. 57 76). London: Routledge.

Centro ESADE de Industrias de la Cultura (2008). El mapa del conocimiento y el 'periodímetro' [The knowledge map and 'periodímetro']. Cuaderno de Periodistas: revista de la Asociación de la Prensa de Madrid, 14(junio), 9-52. Retrieved from http://www.apmadrid.es/wp-content/uploads/images/stories/ doc/vapm20100511132602.pdf

CES (2015). La situación de la I+D+i en España y su incidencia sobre la competitividad y el empleo [The situation of R\&D\&I in Spain and its impact on competitiveness and employment]. Madrid: Consejo Económico y Social de España.

COTEC (2014). Informe Cotec 2014. Tecnología e Innovación en España [Cotec Report 2014. Technology and Innovation in Spain]. Madrid: Fundación Cotec para la Innovación Tecnológica. 
Diezhandino, M. P. (Coord.) (2012). El periodista en la encrucijada [The journalist at a crossroad]. Barcelona: Fundación Telefónica y Ariel.

FECYT (2007). Estrategia Nacional de Ciencia y Tecnología. Comisión Interministerial de Ciencia y Tecnología [National Science and Technology Strategy. Interministerial Commission of Science and Technology]. Madrid: Fundación Española para la Ciencia y la Tecnología.

FECYT (2014). Indicadores del Sistema Español de Ciencia, Tecnología e Investigación 2014 [Indicators of Spanish System of Science, Technology and Research 2014]. Madrid: Fundación Española para la Ciencia y la Tecnología.

Fuente Cobo, C. \& Mera Fernández, M. (2013). Implantación del Método del Caso en la enseñanza del Periodismo en las universidades españolas: un análisis a partir de las guías docentes [Implantation of Case Method in Journalism Studies in Spain. An Analysis Based on Teaching Guides]. Estudios sobre el Mensaje Periodístico, 19 (núm. especial marzo), 177-186. https://doi.org/10.5209/rev_ESMP.2013. v19.42023

Fundación BBVA (2012). Estudio internacional de «Cultura Científica». Comprensión de la Ciencia [International study of «Scientific Culture». Understanding Science]. Madrid: Departamento de Estudios Sociales y Opinión Pública, Fundación BBVA.

García Agustín, J. (2011). La Información Periodística de la Ciencia: Hacia un Modelo de Gestión [The Journalistic Information of Science: Towards a Management Model]. Pamplona: Thomson Reuters Aranzadi.

Giraldo, C. (2015). Ciencia, tecnología y escritura. El encuadre científico de la expresión escrita [Science, technology and writing. The scientific framing of the written expression]. Praxis $\&$ Saber, 6(11), 67-86 https://doi.org/10.19053/22160159.3575

Gutiérrez Rodilla, B. M. (1998). La ciencia empieza en la palabra. Análisis e historia del lenguaje científico [Science begins with words. Analysis and history of scientific language]. Barcelona: Ediciones Península.

Lewenstein, B. (1994). A Survey of Public Communication of Science and Technology Activities in the United States. In B. Schiele (Ed.), When Science Becomes Culture: World Survey of Scientific Culture (pp. 119-178). Boucherville-Quebec: University of Ottawa Press.

Ley 13/1986, de 14 de abril, de Fomento y Coordinación General de la Investigación Científica y Técnica. Boletín Oficial del Estado, núm. 93, de 18 de abril de 198, pp. 13767-13771. Retrieved from https:// www.boe.es/buscar/doc.php?id=BOE-A-1986-9479

Ley 14/2011, de 1 de junio, de la Ciencia, la Tecnología y la Innovación. Boletín Oficial del Estado, núm. 131, de 02 de junio de 2011. Retrieved from https://www.boe.es/buscar/act.php?id=BOE-A-2011-9617

Ley Orgánica 6/2001, de 21 de diciembre, de Universidades. Boletín Oficial del Estado, núm. 307, de 24 de diciembre de 2011. Retrieved from https://www.boe.es/buscar/act.php?id=BOE-A-2001-24515

López Cerezo, J. A. \& Cámara Hurtado, M. (2009). La cultura científica en España [Scientific culture in Spain]. In R. Gutiérrez Rivilla (Coord. Ed.), El español, lengua para la ciencia y la tecnología: presente y futuro [Spanish, language for science and technology: present and future] (pp.17-40). Madrid: Instituto Cervantes.

Mariño, X. (2016, s.f.). Peripeciencia. Discurshow. Message posted to http://www.peripeciencia.nos.gl/ discurshow.html

Méndez Majuelos, I. (2013). Comunicación de la ciencia y la técnica y su impacto en la sociedad. Nuevas fórmulas para un nuevo escenario [Communication of science and technology, and its impact on society. New formula for a new scenario]. In M. Sobrados León (Coord.), Presente y futuro en el Periodismo Especializado [Present and future in Specialized Journalism] (pp. 135-166). Madrid: Fragua.

Meneses Fernández, M. D. \& Martín-Gutiérrez, J. (2015). ¿Tienen razón los investigadores al quejarse de la información periodística sobre ciencias? Experiencias con alumnos de periodismo y científicos [Are researchers right when they complain about media coverage of science? Experiences with journalism students and scientists]. Revista Española de Documentación Científica, 38(4), 104. https://doi.org/10.3989/redc.2015.4.1252 
MINECO (2013). Estrategia Española de Ciencia y Tecnología y de Innovación 2013-2020 [Spanish Strategy for Science and Technology and Innovation 2013-2020]. Madrid: Ministerio de Economía y Competitividad, Gobierno de España.

MINECO (s.f.). Mapa de infraestructuras científicas y técnicas singulares (ICTS) [Map of unique scientific and technical infrastructures]. Retrieved from http://www.idi.mineco.gob.es/stfls/MICINN/Innovacion/ FICHEROS/ICTS_esp.pdf

Moreno Castro, C. (1999). La información científico técnica y la formación democrática de los ciudadanos [Technical scientific information and democratic formation of the citizens.]. Comunicar, (13), 95-100. Retrieved from https://www.revistacomunicar.com/index. php?contenido=detalles \&numero $=13 \&$ articulo $=13-1999-14$

Moreno Castro, C. (2002). Ciencia y tecnología en la formación de los futuros comunicadores [Science and technology in journalists training]. Comunicar, (19), 19-24. Retrieved from https://www.revistacomunicar.com/index.hp?contenido=detalles\&numero=19\&articulo=19-2002-04

Moreno Castro, C. (2003). La investigación universitaria en periodismo científico [University research on scientific journalism.]. Ámbitos. Revista internacional de comunicación, (10), 121-141. Retrieved from http://www.redalyc.org/html/168/16801006/

Rosique Cedillo, G. (2013). Los estudios universitarios en España: la adaptación al EEES y la formación de periodistas en competencias digitales [The university studies of journalism in Spain: the adaptation to the EHEA and the training of journalists in digital skills]. Revista Internacional de Estudios sobre Sistemas Educativos, 1(1-2), 117-132. Retrieved from http://www.riesed.org/index.php/ RIESED/article/view/12

Sánchez-Mora, M. C. (2016). Hacia una taxonomía de las actividades de comunicación pública de la ciencia [Towards a taxonomy for public communication of science activities]. Journal of Science Communication, 15(02). Retrieved from https://jcom.sissa.it/sites/default/files/documents/ JCOM_1502_2016_Y01_es.pdf

Trelles Rodríguez, I. \& Rodríguez Betancourt, M. (2013). Comunicación de la Ciencia y la Tecnología y cultura científica para la prevención de riesgos. Experiencia cubana [Scientific communication and scientific culture for risk prevention. Cuban experiences]. MHCJ, 4, 213-231. Retrieved from http://rev.innovacionumh.es/index.php?journal=mhcj\&page $=\operatorname{article} \& o p=v i e w \& p a t h \% 5 \mathrm{~B} \% 5 \mathrm{D}=10 \&$ path\%5B $\% 5 \mathrm{D}=19$

\section{SOBRE LAS AUTORAS}

María Dolores Meneses Fernández, licenciada y doctora en Ciencias de la Información y licenciada y doctora en Geografía e Historia. Profesora de Periodismo en la Universidad de La Laguna (España). Su docencia e investigación se centra en Periodismo Especializado y Deontología de la Comunicación. Uno de sus temas de investigación es la aplicación de la realidad aumentada a la narrativa periodística. Es autora de numerosas publicaciones académicas entre libros, capítulos de libros y artículos sobre Comunicación e Historia.

Yasmina Rivero Abreu, graduada en Periodismo y licenciada en Geografía e Historia. Posee un magíster en Comunicación Científica. Es técnico superior de la Unidad de Cultura Científica y de la Innovación de la Universidad de La Laguna y técnico de biblioteca pública. Es profesora en el sistema canario público de educación secundaria. 